\title{
Aproximación al desarrollo rural desde la nueva ruralidad
}

\section{The rural development: An approach from the New rurality}

Fecha de recepción: 1 de septiembre de 2014 Fecha de aceptación: 20 de noviembre de 2014
Luz Adriana Pita-Morales' ${ }^{1}$ Wilson Gonzalez-Santos² Elnar Daniel Segura-Laiton ${ }^{3}$

\section{Resumen}

El desarrollo rural ha sido objeto de distintas aproximaciones conceptuales, en función del momento socioeconómico espacial imperante; se destacan el estructuralismo y la modernización, que tuvieron influencia desde la década de los cincuenta hasta mediados de la de los sesenta; la dependencia, desde mediados de los años sesenta y hasta el final de los setenta; el neoliberalismo, desde los ochenta; el neoestructuralismo y las estrategias de vida rural, a partir de los noventa (1), y el enfoque territorial (2). La nueva ruralidad, que puede ubicarse en el enfoque de estrategias de vida, ha tenido un amplio desarrollo y estudio en América Latina; con esta orientación se intenta entender la complejidad y la dinámica del espacio rural, que trasciende su tradicional concepción de sector productor y abastecedor de alimentos; dentro de este marco es pertinente su estudio, si se tiene en cuenta que el contexto rural es precisamente el nicho de trabajo de los profesionales del sector agrario. Con esta revisión se pretende contribuir a la construcción del concepto de nueva ruralidad, como herramienta para el análisis del sector rural, a través de la compilación y análisis de los aportes de diferentes autores. Los resultados indican que este enfoque se corresponde con la adaptación de la población rural a fenómenos económicos, sociales y culturales, reconociendo que la multidimensionalidad del espacio rural es una aproximación pertinente que permite comprender e intervenir en los problemas del desarrollo rural.

Palabras clave: Desarrollo rural, Nueva ruralidad, Territorio.

\begin{abstract}
The rural development has been subject to different conceptual approximations, in function of the prevailing socio-spatial time. There stands out: the structuralism and the modernization which had

1 M.Sc. Universidad de Boyacá (Tunja-Boyacá, Colombia).

2 Ph.D. (c) Universidad Pedagogica y Tecnologica de Colombia (Tunja-Boyacá, Colombia).wilson.gonzalez@uptc.edu.co.

3 Profesional Independiente.
\end{abstract}


influence from the 50's to the mid 60's, the dependence from the mid 60's until the end of $70^{\prime} \mathrm{s}$, the neoliberalism since the $80^{\prime} \mathrm{s}$, the neostructuralism and the rural life strategies starting from the 90's (1) and the territorial approach (2). The new rurality can be located on the life strategies approach and it has had a broad development and study in Latin America. With this orientation is intended to understand the rural areas complexity and dynamics, which transcends its traditional conception as a producer and food supplier sector. This frame work is pertaining to study, due that the rural context, is the agrarian professionals' work place.

With this review it is pretended to contribute to build the new rural scene concept, as a tool to analyze the rural sector by compiling and analyzing the different authors contributions. The results indicate that this approach corresponds to the rural population adaptation to the economic, social and cultural phenomena, recognizing the multidimensionality of rural areas, is a relevant approach to understand and to intervene in the rural development problems.

Keywords: Rural Development, New Rurality, Territory. 


\section{Introducción}

La importancia del medio rural, tanto como despensa alimentaria como fuente ambiental fundamental, contrasta con la heterogeneidad de su población (3).En Colombia, el $68 \%$ de la población rural es pobre ${ }^{4}$, y el $27,5 \%$ vive en la miseria; además, presenta, entre otras características, analfabetismo, alto número de integrantes dependientes del jefe de hogar $y$ empleo formal escaso. A pesar de que el $74 \%$ de los jefes de hogar están vinculados a la agricultura, los salarios de empleos informales y de actividades no agrícolas son los que sostienen el $77 \%$ de estos hogares (4); de acuerdo con lo anterior, las labores agrícolas, aunque son importantes en el ámbito rural, no son suficientes para explicar la totalidad del ingreso de la población que habita en este medio.

En pro de solucionar la crisis del campo, el Estado colombiano ha adoptado diversos modelos de desarrollo; sus apuestas han girado entre una agricultura comercial y exportadora y una agricultura campesina tradicional $^{5}$, soslayando la confusión derivada de las relaciones y particularidades que caracterizan las dinámicas territoriales del medio rural y que se convierte en el problema por resolver con la presente revisión.

Esta revisión documental hace parte de la descripción protocolar del proyecto "El desarrollo rural contemporáneo en la educación en las ciencias agrarias: un estudio de caso en la Universidad Pedagógica y Tecnológica de Colombia". A partir de la Nueva Ruralidad, la revisión tiene como objetivo aportar elementos conceptuales que permitan una intervención pertinente en el espacio rural.

4 Desde la perspectiva del ingreso: población con ingresos menores de US\$1 diario (5). Informes preliminares del tercer censo agropecuario realizado en Colombia incluyen el Índice de pobreza multidimensional ajustado, que reporta un valor de 44,7\% para el medio rural en el 2014 (6).

5 Aunque las dos pueden coexistir en un mismo espacio, lo relevante es la complejidad de las interacciones resultantes de la yuxtaposición de sus actores (7).

\section{Metodología}

Siguiendo los elementos propuestos por la Nueva Ruralidad, se procedió a realizar una investigación documental de tipo descriptivo (8); posteriormente, y en concordancia con Roussos (9), la investigación desarrolló las siguientes etapas:

- Definición correcta del tema: consistió en generar un aporte a la actualización conceptual de los fenómenos socio-económicos y culturales del medio rural.

- Búsqueda bibliográfica: selección de principales autores, consulta de bases de datos y fuentes documentales, acceso a principales documentos.

- Análisis de los documentos: con el programa Atlasti. 6 se realizó la extracción, categorización y entrelazamiento de los segmentos comunes para la revisión.

- Síntesis de la información: en esta etapa se construyó un mapa conceptual que presenta las principales temáticas que abarca el concepto de Nueva Ruralidad.

El medio rural ha recibido aportes de diferentes corrientes: el estructuralismo y la modernización, la dependencia, el neoliberalismo y el neoestructuralismo, las estrategias de vida rural y el enfoque territorial. Kay (1) ubica la Nueva Ruralidad en las estrategias de vida, cuyo origen es británico, pero ha tenido un amplio desarrollo y estudio en América Latina; este enfoque surge como una respuesta al cambio de estrategias desarrollistas provenientes del estructuralismo y el neoliberalismo.

De esta manera, de tener, de los años cincuenta a los setenta, un modelo de desarrollo basado en la ampliación de la frontera agrícola, en la transferencia de tecnología, en la construcción de mercados internos, en los incentivos a la agricultura, en la inversión directa al campo y en los subsidios a la producción (10) se pasó, a partir de los años ochenta, a un modelo de apertura económica y liberalización de las fronteras 
económicas (11).Siguiendo a Llambi (11), como consecuencia de este proceso hubo incremento de la pobreza rural, afectación del medioambiente y desigualdad económica y cultural en el mundo rural, el Estado y las naciones.

Los efectos de la aplicación del nuevo modelo no han sido uniformes; mientras algunos sectores han sido beneficiados y han resultado más competitivos, los objetivos que se dice persigue la globalización, en términos de bienestar, equidad y progreso, no se han cumplido. Los niveles de pobreza son altos, el aumento de la inequidad en la distribución es evidente, los niveles de crecimiento no son sostenibles (12).

La globalización es un proceso de restructuración de relaciones económicas, sociales y culturales, donde se comprometen e impactan, entre otros aspectos propios de las regiones y naciones: la depredación del medioambiente, la desintegración de las entidades culturales, la apropiación de la naturaleza y la desarticulación de la economía campesina (13). En respuesta a estos fenómenos y procesos, surge la Nueva Ruralidad, como una posible acomodación de la población residual del fenómeno neoliberal. Se originan nuevos movimientos indígenas y campesinos, forjando ideas e identidades para sobrevivir (14).

La nueva ruralidad es un proceso de cambio, producto de la globalización del capital, resultado de los proyectos de organización de la vida de los sujetos sociales que se resisten o se relegan de esta tendencia mundial (13). De esta manera, la lucha constante y la transformación de los campesinos e indígenas por sobrevivir en sus diferentes formas de vida da origen a una "nueva ruralidad", donde la exigencia por obtener mejores precios para sus productos, mayor acceso a tierras, créditos y asistencia técnica originan que el mero concepto de producción agrícola sea abolido y se creen nuevas estrategias de vida. Grammont (15) pone en evidencia algunos fenómenos que tratan de explicar la nueva ruralidad-entre otrosrelacionados con corregir la brecha existente entre el campo y la ciudad, la urbanización del campo y la ruralización de la ciudad y las tecnologías revolucionarias de la vida en el campo y la ciudad.
Una nueva relación campo-ciudad en donde los límites entre ambos ámbitos se desdibujan, se confunden y se complejizan.

Para otros autores, la nueva ruralidad se desarrolla en zonas cuya población realiza actividades enmarcadas en diversos sectores como agricultura, artesanía, ganadería, industrias pequeñas y medianas, comercio, servicio, pesca y minería, entre otros (16). Se analizan dos aspectos dentro de este concepto: el relacionado con las particularidades históricas, sociales, culturales y ecológicas, que tiene una realidad propia, como lugar de vida, y aquel que se concentra con la tenencia de la tierra y de otros recursos naturales (17). Llambí (18) afirma que la nueva ruralidad se refiere a las reestructuraciones geoeconómicas y a reacomodos geopolíticos en diferentes niveles (global, nacional y local), señalando tres aspectos de intervención: la contra urbanización, la transformación hacia actividades económicas terciarias y secundarias y el cambio de estilos de vida dados por la modernidad.

Enseguida se presentan los aportes de distintos autores al concepto de nueva ruralidad, en función de las categorías heterogeneidad histórica, heterogeneidad productiva, multifuncionalidad y territorio.

\section{Heterogeneidad histórica}

Trata lo rural de acuerdo con la actividad económica tradicional: la ocupación agrícola. El concepto de nueva ruralidad data de los comienzos de 1990, y se planteó con el fin de dar explicación a las relaciones entre los macroprocesos globales y los procesos territoriales que estaban sucediendo en ese momento (19); para algunos autores este concepto identifica nuevos fenómenos y procesos, y otros opinan que es un nuevo enfoque para describir e interpretar los viejos procesos de cambio del mundo rural (20).

Llambi y Pérez (21) refieren que la nueva ruralidad se concentra en los procesos que tienen lugar a diferentes escalas (global, nacional y local), involucrando los centros poblados dispersos. La ruralidad ha estado ligada, principalmente, a tres 
características: una baja densidad demográfica, el predominio de la agricultura en la estructura productiva y económica, y unos rasgos fundamentados en los valores, las creencias y las conductas. A lo anterior hay que sumar el cambio en el paisaje rural, que ha sido visto como campos cultivados o, en algunas ocasiones, como ecosistemas deshabitados. En la actualidad, extensiones de cultivos bajo invernadero, industrias de lácteos, explotaciones de pollos y empresas de transformación de la materia prima, entre otras infraestructuras, hacen cambiar totalmente el aspecto rural (22).

Un nuevo paisaje rural genera cambios de tipo estructural, explicados por nuevas relaciones entre la población y su espacio, que se derivan en nuevos territorios. Algunas actividades que caracterizan esta ruralidad son el transporte, los servicios básicos y la educación (19). Jiménez (23) plantea un ejercicio en el que con cuestionamientos comparativos define lo rural y lo urbano: lo rural excluye lo urbano y viceversa, en un círculo vicioso cada concepto se liga con el otro con puntos de contacto comunes. Lo complejo se presenta cuando en lo rural, la mayoría de empleos e ingresos provienen de actividades no agrícolas (21).

Gómez (24) muestra algunas características que definen una zona como tradicional rural: la población se ocupa en actividades agropecuarias, fenómenos naturales intervienen en la explotación con un grado menor de control por parte del hombre, la población se ubica en baja densidad y refleja condiciones culturales atrasadas $y$ presencia de analfabetismo, atraso material y bajo bienestar. En esta línea, Nisbet (25) plantea que el conservadurismo representa la sociedad local o rural, mientras que el racionalismo representa lo moderno o urbano. Gómez (24) caracteriza los dos espacios en función de algunas características (Tabla I).

Tabla I. Concepto tradicional moderno.

\begin{tabular}{cc}
\hline Tradicional & Moderno \\
\hline Campo & Ciudad \\
Agricultura & Industria \\
Rural & Urbano \\
\hline
\end{tabular}

El vocablo rural concierne al ser humano y a su medio, a sus múltiples relaciones y al conjunto de sus actividades, es decir, ha tenido una relación más socio-antropológica que productivista, la cual envuelve aspectos relacionados también con la salud, la educación, la vivienda, la dotación de servicios básicos, el patrimonio cultural, las redes sociales y el ejercicio de la ciudadanía (26). Esta percepción tiende a separar el concepto rural de lo agropecuario, ya que el primero implica tener en cuenta la multidimensionalidad ocurrente en el quehacer cotidiano del ser humano. Zimmerman y Sorokin, citados por Sevilla (27), definen variables que diferencian lo urbano de lo rural (Tabla II).

Tabla II. Dimensiones e indicadores en el análisis rural-urbano.

\begin{tabular}{cc}
\hline Dimensiones & \multicolumn{1}{c}{ Indicadores } \\
\hline Productiva & Naturaleza de la actividad económica, fuentes de ingreso. \\
Ambiental & Transformación del ambiente natural. \\
Demográfica & Número de personas por unidad de superficie, tamaño de los centros poblados. \\
Social & División del trabajo, ingresos, tiempo de permanencia, diferenciación ocupacional. \\
Cultural & Cambio sociocultural y cosmopolitismo.
\end{tabular}

Finalmente, para González (7) la nueva ruralidad estudia las relaciones que emergen entre los espacios rural-urbanos, redefiniendo el concepto de espacio rural residual, dada la reciprocidad de la relación rural-urbana.

Heterogeneidad productiva 
Se refleja en el surgimiento o incremento de una variedad de actividades distintas a la actividad agrícola (13).Se ha denominado ocupacional, pluriactividad, desacralización de lo rural, multiocupación o multiactividad. Para Mascheroni y Riella (28), la pluriactividad es la combinación en un individuo o en una familia de actividades agrícolas y no agrícolas. Hace énfasis en la visión de territorio, donde constantemente se combinan y articulan, en diferentes proporciones y modalidades, actividades rurales con urbanas.

El crecimiento de las actividades no agrícolas en el medio rural puede estar relacionado con la demanda en los mercados del trabajo rural, influyendo en los nuevos modelos de ocupación de las fuerzas de trabajo y generando el aumento de la presencia de actividades no agrícolas en la zona rural o sus alrededores (29).

El concepto de "no agrícola" pone de manifiesto el desarrollo de actividades del sector secundario, la industria y la manufactura, así como los servicios, y excluye la producción primaria de productos agroalimentarios con base en uno o más factores de producción que corresponden a los recursos naturales (30). Se considera que el empleo rural no agrícola (ERNA) da solución a algunos problemas del sector, como la pobreza y la escasa agregación de valor. Según la Cepal (31), los hogares de bajos recursos ven una salida en el empleo rural no agrícola, no solo para mejorar sus ingresos, sino para tener una estabilidad económica durante todo el año.

Para Berdegué et al. (30), el ERNA se origina por la modernización del espacio rural: entre más moderno y competitivo el sector rural, mayor será su industrialización. De esta manera, un desarrollo in situ trae consigo bienestar social. En las actividades de la nueva oferta del sector rural se destacan, entre otras, la agroindustria rural, el turismo rural y el agroturismo, además de los aportes que estas actividades realizan al mantenimiento del medioambiente y los recursos del territorio (13).

En otro estudio, Berdegué et al. (32) relacionan la tipología del empleo rural con vínculos de producción con la agricultura y con actividades primarias no agrícolas, vinculadas al consumo de la población rural y urbana y a los servicios públicos.

La pluriactividad y otras transformaciones económicas, demográficas e institucionales han sido el resultado de procesos de industrialización (16) que condujeron a una aglomeración urbana y al desarrollo tecnológico, optimizando el uso de los factores de producción, principalmente de mano de obra y de tierra.

Entre tanto, para Teubal (33) la multiocupación constituye una manifestación más de la exclusión social de la globalización en el medio rural; este fenómeno trae consigo otros efectos, como la precarización del empleo rural, la expulsión de pequeños y medianos productores y la migración del campo a la ciudad. De esta manera, la naturaleza del empleo en lo rural cambia desde su origen, relacionado con lo agrícola y pecuario, al alquiler de mano de obra en el comercio, la industria y el turismo: empleo rural no agrícola (ERNA).

Para Kay (14), el ERNA derivado de la multiocupación tiene un significado diferente para cada unidad familiar, dependiendo de su nivel económico y del acceso a los factores de producción. Para los pobres es una manera de subsistencia e inyección de capital a sus parcelas, mientras que para los ricos es una manera de aumentar el capital.

Estudios en Honduras (34) y Ecuador (35) señalan algunos efectos del ERNA sobre la pobreza, la seguridad alimentaria y la distribución del ingreso rural. La integración del ERNA en lo rural permite una interpretación integral de ruralidad (36).

\section{Multifuncionalidad de la agricultura}

El concepto de multifuncionalidad tiene sus soportes en la Cumbre de la Tierra (37), y promueve que el desarrollo no excluye la sostenibilidad y la protección al medioambiente. La Organización para la Cooperación y el Desarrollo -OCDE- (38) puntualiza que, además de su función primaria de 
producir alimentos y fibras, la actividad agrícola provee múltiples beneficios tanto ambientales como económicos y sociales (39):

- Económicos: la agricultura como fuerza de sostenimiento y crecimiento de la economía, incluso en los países altamente industrializados (40).

- Sociales: el momento social favorece el desarrollo de actividades económicas (41).

- Ambientales: la protección y conservación de los recursos naturales de una región, como un bien común que provee a la comunidad beneficios para la vida (42).

La multifuncionalidad de lo rural se presenta como respuesta a las demandas que se generan desde lo urbano, de las relaciones y la conexión entre lo rural y lo urbano (13).Se refiere a los productos, servicios y externalidades derivados de la actividad agrícola que tienen impacto sobre la economía y la sociedad y tienen la cualidad de ser positivas, limitando las externalidades negativas de la agricultura (10).En el concepto hay dos corrientes:

1. La multifuncionalidad positiva: corrige las externalidades negativas a nivel ambiental; da un valor más allá del económico y de mercado a los productos agrícolas (10).

2. La multifuncionalidad normativa: explica las contribuciones de la agricultura al desarrollo económico y social en términos de las externalidades positivas que son reconocidas por la sociedad (43).

La multifuncionalidad redefine al mismo tiempo las ventajas y desventajas comparativas de los territorios rurales, en el contexto de los mercados mundiales (11). Este auge de nuevas funciones del espacio rural ha contribuido a cerrar la brecha entre lo rural y lo urbano (44).

El Instituto Interamericano de Cooperación para la Agricultura -IICA- (45) afirma que el medio rural tiene nuevas demandas vinculadas a los recursos endógenos, la diversidad y los recursos naturales.
En tal sentido, la multifuncionalidad rescata la importancia de los espacios de reserva ecológica, favoreciendo la sustentabilidad del ecosistema (46).

En este marco es necesario conocer la oferta multifuncional de cada sistema agrario para dar una correcta manipulación de la oferta y la demanda que surgen de la sociedad (47), lo que ha permitido en algunos estudios adoptar otro tipo de conceptos: i) la multifuncionalidad sectorial, que aborda las funciones derivadas de la producción agrícola por las que el agricultor no recibe ningún tipo de pago, y ii) la multifuncionalidad territorial, que entiende el medio rural como un territorio dotado de recursos específicos $(48,49)$.

\section{Territorio}

En los últimos años, el territorio, sus características y cualidades se han convertido en un factor importante para el desarrollo. Se ha planteado como estrategia de desarrollo el enfoque territorial (50). En este escenario, el concepto de territorio es un constructo social: un espacio geográfico con una identidad social, económica y cultural. A partir de estas características, un territorio se puede construir tipológicamente interpretando sus fortalezas y debilidades como bitácora para su desarrollo (51).

El desarrollo territorial involucra al individuo en su propio proceso de desarrollo, de acuerdo con las ventajas que ofrece su territorio. La OCDE (39) indica que el desarrollo genera un aumento de bienestar en la población de un territorio. En este sentido se deben tener en cuenta tres factores que definen sus características: recursos específicos, actores territoriales y acuerdos institucionales. A partir de este planteamiento toman valor las diferencias entre el ecosistema, la historia, la cultura, las costumbres y tradiciones, la arquitectura institucional, la población con sus conocimientos y las habilidades (52).

Para este enfoque, el territorio no es un mero espacio hermético, sino una construcción social; de esta manera, no puede haber una definición conceptual definitiva genérica; para cada proceso 
de desarrollo rural, es el espacio que sus agentes reconocen como necesario y propio, en el cual se delimitan las relaciones, en su interior y su exterior (53). Con lo anterior, los territorios encuentran una fortaleza en su desarrollo al ser diversificados y tener una identidad para conquistar mercados especializados (54).

La Tabla III sintetiza las características de las temáticas que orientaron el desarrollo del presente ejercicio.

Tabla III. Temáticas en la nueva ruralidad.

\begin{tabular}{cc}
\hline Temática & Características \\
\hline Antecedentes & Transformación de las teorías de desarrollo \\
Heterogeneidad productiva & Actividades diversas a la producción agrícola en lo rural. \\
Heterogeneidad histórica & Revaloriza el campo. Desdibuja la brecha campo-ciudad. \\
Multifuncionalidad & La agricultura trasciende la mera producción de alimentos. \\
Territorio & El territorio como una construcción social. \\
\hline
\end{tabular}

La Fig. 1 ilustra el surgimiento de la nueva ruralidad y de las principales temáticas que aborda. La interacción medioambiente, espacio territorio y componente social definen las características de lo que se ha denominado Nueva Ruralidad.

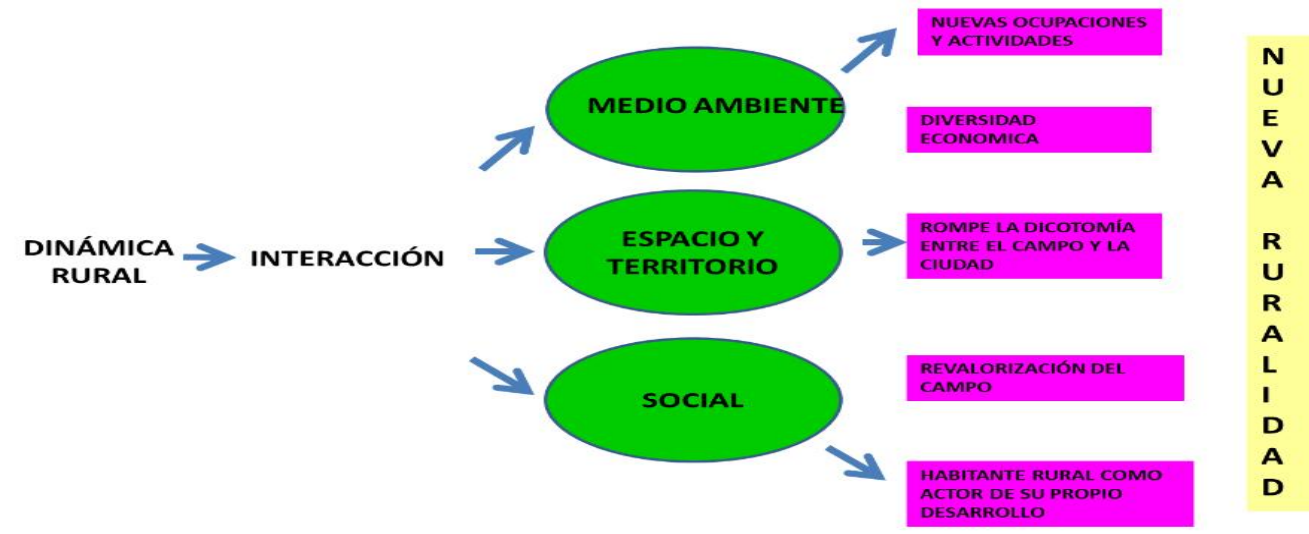

Figura 1. La Nueva Ruralidad.

\section{Conclusiones}

- El desarrollo rural ha sufrido cambios producto de los enfoques económicos de desarrollo imperantes en el mundo; dichos cambios se relacionan con trasformaciones de las actividades económicas que se realizan en el medio rural y con la revalorización de los recursos territoriales del campo, que promueven un desarrollo rural basado en el aprovechamiento de las ventajas con que cuentan los territorios rurales.
- Es de vital importancia la actualización teórica sobre los fenómenos que suceden en el medio rural y que demarcan su desarrollo, debido a que es un medio que está en constante cambio y evolución, el cual, si se pretende intervenir, se debe conocer, con el fin de realizar aportes positivos y en concordancia con el momento que se desenvuelve.

- El desarrollo rural ya no está regido solamente por actividades netamente agropecuarias, por tal motivo es importante que en la formación de profesionales que trabajarán como 
promotores del desarrollo rural se involucre la complejidad del medio rural a fin de una intervención adecuada en dicho espacio.

\section{Referencias}

(1) Kay C. Enfoques sobre el Desarrollo Rural en América Latina y Europa desde Mediados del Siglo XX. JAVEGRAF. Bogotá. 2007.

(2) RIMISP, Centro Latinoamericano para el Desarrollo Rural. Estrategia de Implementación del Programa de Desarrollo Rural Integral con Enfoque Territorial. Departamento Nacional de Planeación. Bogotá. 2014.

(3) Pérez E. y Pérez M. El sector rural en Colombia y su crisis actual. Cuadernos de Desarrollo Rural, 2002; 48(35): 58-65.

(4) Centro de Estudios Regionales Cafeteros y Empresariales. Pobreza rural: evaluación y diagnóstico de las políticas nacionales. Departamento Nacional de Planeación. Bogotá. 2007.

(5) Banco Mundial (en línea). Definición de los objetivos de desarrollo del milenio, pobreza extrema y hambruna. 2012 (fecha de acceso 11 de abril de 2012); Disponible en: www. bancomundial.org.

(6) El Espectador. Definición de los objetivos de desarrollo del milenio. Pobreza extrema y hambruna. Redacción económica (en línea),2015 (fecha de acceso 10 de octubre de 2015). Disponible en: http://www.elespectador.com/tags/censo-agropecuario.

(7) González W. Educación y entorno en la ingeniería agronómica, un encuentro entre la academia y su contexto. Editorial Universidad Pedagógica y Tecnológica de Colombia. Tunja. 2009.

(8) Guirao G., Salas O. y Ferrer F. El artículo de revisión. Rev. Iber. de enfe. Comu. (en línea), 2008. (fecha de acceso 08 de Enero de 2014). Disponible en:http://revista.enfermeriacomunitaria.org/articulo.
(9) Roussos A. Preparación de una revisión bibliográfica para su publicación, cuando un solo artículo nos habla de muchos trabajos. Fund para la inve en cien soci.2011; 1(7):1-7 p.

(10) Bonnal P., Bosc J., Díaz M.,Losch B. Multifuncionalidad de la agricultura y nueva ruralidad ¿Restructuración de las políticas públicas a la hora de la globalización? JAVEGRAF. Bogotá. 2004.

(11) Llambi L. Nueva Ruralidad, Multifuncionalidad de los espacios rurales y Desarrollo Local Endógeno. JAVEGRAF. Bogotá. 2004.

(12) Echeverri R., Ribero P. Nueva ruralidad, Visión del territorio en América Latina y el Caribe. IICA. Costa Rica. 2002.

(13) Concheiro L.,Ochoa K.,GrajalesS. Nueva ruralidad. Enfoques y propuestas para América Latina. Centro de estudios para el desarrollo rural sustentable y la soberanía alimentaria. Cámara de diputados. México. 2006.

(14) Kay C. Estrategias de vida y perspectivas del campesinado en América Latina. ALASRU. México. 2005.

(15) Grammont H. El concepto de nueva ruralidad. JAVEGRAF. Bogotá. 2008.

(16) Pérez E. Hacia una nueva visión de lo rural. JAVEGRAF. Bogotá. 2001.

(17) Baudel M. ¿Una nueva ruralidad en América Latina? CLASCO. Buenos Aires. 2001.

(18) Llambí L. Globalización, Ajustes y Nueva Ruralidad: Una Agenda para la Investigación y el Desarrollo Rural. JAVEGRAF. Bogotá. 1995.

(19) Pérez E., Farah M. La Nueva Ruralidad en Colombia. JAVEGRAF. Bogotá. 2006.

(20) Riella A., Romero J. Nueva Ruralidad y empleo-no agrícola en Uruguay. Territorios y Organización social de la agricultura. Buenos Aires. 2003. 
(21) Llambi L. y Pérez E. Viejas ruralidades y nuevos campesinismos, Agenda para una nueva sociología rural latinoamericana. Cuad. de desa. rural.2007; pp. 37-61.

(22) Friedland W. Agriculture and rurality: beginning the final separation? Rural Sociology. 2002; 67: 350-371.

(23) Jiménez C. La articulación entre lo rural y lo urbano. Problema teórico y metodológico de la sociología rural. ALASRU. México 2011.

(24) Gómez S. ¿Nueva Ruralidad? Un aporte al debate. Rev Soci y Agri. 2001; 17(1): 5-32.

(25) Nisbet R. Las Ideas, Unidades de la Sociología. HICITEC. Brasil 1981.

(26) Suárez N. y Tobasura I. Lo Rural. Un Campo Inacabado.Revi Facu Naci Agro Mede.2008; 61(2): 80-95.

(27) Sevilla E. De la sociología rural a la agroecología. Icaria. Barcelona. 2006.

(28) Mascheroni P., Riella A. Evidencias empíricas sobre la pertinencia de la nueva ruralidad en Uruguay. JAVEGRAF. Bogotá 2003.

(29) Schneider S. La contribución de la pluriactividad para las políticas públicas de desarrollo rural: una mirada desde el Brasil. ALASRU. Quito. 2007.

(30) Berdegué J.,Reardon T., Escobar G. La creciente importancia del empleo y el ingreso rurales no agrícolas. BID. Washington. 2001.

(31) CEPAL. Empleo rural no agrícola y pobreza en América Latina: Tendencias recientes. Naciones Unidas. Chile. 2004.

(32) Berdegué J., Reardon T., Escobar G. Opciones para el desarrollo del empleo rural no agrícola en América Latina. BID. Washington. 2001.
(33) Teubal M. Globalización y Nueva Ruralidad en América Latina. CLASCO. Buenos Aires. 2001.

(34) Rubén R.,Van Den Berg M. 2004. Empleo no agrícola y alivio de la pobreza de los hogares rurales de Honduras. CEPAL. Santiago 2001.

(35) Elbers C., Lanjouw P. Transferencia intersectorial, crecimiento y desigualdad en Ecuador rural. CEPAL. Santiago. 2004.

(36) Echeverri R. La Nueva Ruralidad. JAVEGRAF. Bogotá. 2001.

(37) FAO. Lucha contra la contaminación agrícola de los recursos hídricos. FAO. Roma. 1997.

(38) Organización para la Cooperación y el Desarrollo. Desempeño ambiental de la agricultura en la OCDE. Paris 2008.

(39) Organización para la Cooperación y el Desarrollo. La réforme de la politiqueagricole et l'économieruraledans les pays de I'OCDE. OCDE. Paris. 1998.

(40) Van G., Vandermeulen V., Mettepenningen E., VerspechtA. Multifunctionality of Agriculture: A Review of Definitions, Evidence and Instruments. ASHGATE 2007.

(41) Grifón M. La multifonctionnalité de I'agriculture, définitions, exemples, enjeux. $\mathrm{Cl}$ RAD-TERA. Paris. 2004.

(42) Rodríguez G. La multifuncionalidad de los sistemas agroalimentarios locales en países en desarrollo: El caso de la agroindustria panelera colombiana. Pers Rura Univ Naci. 2008; 1(2): 113-125.

(43) Laurent C. Activité agricole, multifonctionnalité, pluralité. CTE. Paris 1999.

(44) Link T. El campo en la ciudad: reflexiones en torno a las rurales emergentes. JAVEGRAF. Bogotá 2001. 
(45) IICA. El desarrollo rural sostenible en el marco de una nueva ruralidad. IICA. Panamá 2000.

(46) Ramírez V., Blanca R. La vieja agricultura y la nueva ruralidad: enfoque y categorías desde el urbanismo y la sociología rural. UAM-A. México. 2003.

(47) Arjona M., Calatrava J., Ceña F., Gallardo R., Guijarro R., Millán C.et al. Multifuncionalidad agraria, desarrollo rural y políticas públicas: nuevos desafíos para la agricultura. Consejería de agricultura y pesca. Sevilla. 2009.

(48) Van H., Durand G. Multifunctional Agriculture: A new Paradigm for European Agriculture and Rural Development. ASHGATE. 2003.

(49) Renting H., Rossing W.A.H., Groot J.C.J., Van der Ploeg J.D., Laurent C., Perraudd D. Exploring multifunctional agriculture. A review of conceptual approaches and prospects for an integrative transitional framework. Journal of Environmental. 2009. 90: 112-123.
(50) Kay C. Estudios rurales en América Latina en el periodo de globalización neoliberal: ¿Una Nueva Ruralidad? Revi Mexi de Soci. 2009;71(4): 607-645.

(51) Schejtman A.,Berdegué J. Towards a Territorial Approach for Rural Development. Icaria. Barcelona 2008.

(52) Miranda C., Renault A. Sustainable Development and the Territorial Approach: Identities and Typologies. IICA. Panamá 2007.

(53) Albanesi R., Preda G. El enfoque territorial como propuesta de intervención para el desarrollo. Reflexiones desde una perspectiva latinoamericana. JAVEGRAF. Bogotá. 2005.

(54) Balanza P. Visiones sobre la territorialidad y el desarrollo rural (en línea) 2004 (fecha de acceso 06 de marzo de 2013). Disponible en: http://www.rimisp.org/boletines/bol25/. 